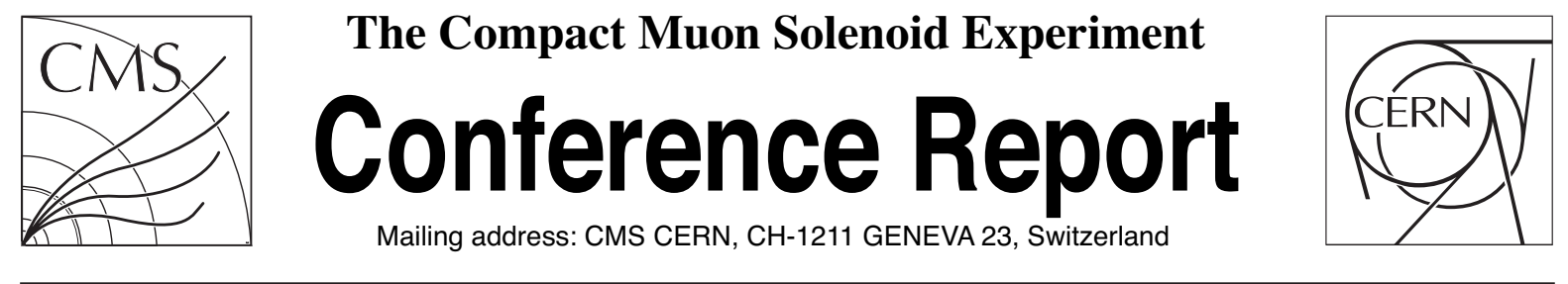

9 November 2007

\title{
The Simulation of the CMS Silicon Tracker
}

\author{
Riccardo Ranieri \\ CERN, Geneva, Switzerland
}

\begin{abstract}
The CMS Tracker is the largest all silicon tracking detector ever built. It consists of $201 \mathrm{~m}^{2}$ of silicon strip detectors, already assembled, and $1 \mathrm{~m}^{2}$ of silicon pixel detectors expected to be assembled by Fall 2007. A total of 1440 pixel and 15148 microstrip modules are mounted in several substructures for a total of about 66 million and 9 million readout channels, respectively.

The physics analysis potential of CMS depends on the accuracy of the simulation of such a complex device. The fast shaping times of the electronics and the signal degradation due to the radiation damage caused by the large flux of particles produced in the LHC collisions are a challenge for the simulation. The accuracy of the predicted space point resolution depends on the ability to correctly model several factors: the diffusion of charges in the $4 \mathrm{~T}$ magnetic field of CMS, the delta ray emission, the inter-strip capacitance, the collected charge and the noise. In addition, a detailed description of the positioning of the detectors is demanded.

The large number of channels with locally mounted electronics requires power and cooling. The large amount of passive material causes multiple scattering, nuclear interactions, electron bremsstrahlung and photon conversions, whose precise simulation demands an accurate evaluation of the passive material budget.
\end{abstract}

Presented at Presented at the 2007 Nuclear Science Symposium and Medical Imaging Conference, Honolulu, Hawaii, $27^{\text {th }}$ October- $3^{\text {rd }}$ November 2007. 


\section{The CMS All-Silicon Tracker}

The silicon Tracker [1] of the CMS experiment [2] at LHC [3] is the largest silicon tracking detector ever built. It consists of a pixel vertex detector and several layers of microstrip silicon detectors.

The pixel detector is a fundamental device for b-tagging studies and impact parameter measurements. It has also paramount importance as a starting point in reconstructing charged particle tracks. It covers the pseudorapidity region $|\eta|<2.4$ and it is organized into three $53 \mathrm{~cm}$ long barrel layers (Pixel Barrel=PXB), positioned at radii of 4.4, 7.3 and $10.2 \mathrm{~cm}$, and two disks per each side (Pixel Forward=PXF), placed at $\pm 34.5 \mathrm{~cm}$ and $\pm 46.5 \mathrm{~cm}$ from the nominal interaction point, covering radii between 6 and $15 \mathrm{~cm}$ to guarantee at least two crossed layers per charged particle track. The pixels have a size of $100 \times 150 \mu \mathrm{m}^{2}$ and are combined with analog signal readout to profit of charge sharing effects and improve the position resolution by interpolation.

The silicon microstrip tracker covers a tracking volume up to a radius of $1.2 \mathrm{~m}$ with a length of $5.6 \mathrm{~m}$ and is organized in three parts: the inner tracker with 4 barrel layers (Tracker Inner Barrel=TIB) and 3 disks per endcap (Tracker Inner Disks=TID), 6 outer barrel layers (Tracker Outer Barrel=TOB) closed by 9 wheels on both sides (Tracker End-Cap=TEC).

The shape of the modules is rectangular in the barrel with the strips parallel to the beam direction for $\varphi$ and $r$ coordinate measurements. The endcap modules are trapezoidal-shaped (wedge-shaped) to allow a radial strip disposition with respect to the beam axis for $\varphi$ and $z$ measurements. The layout of the CMS Tracker is shown in Fig. 1, double lines indicate the double-sided modules.

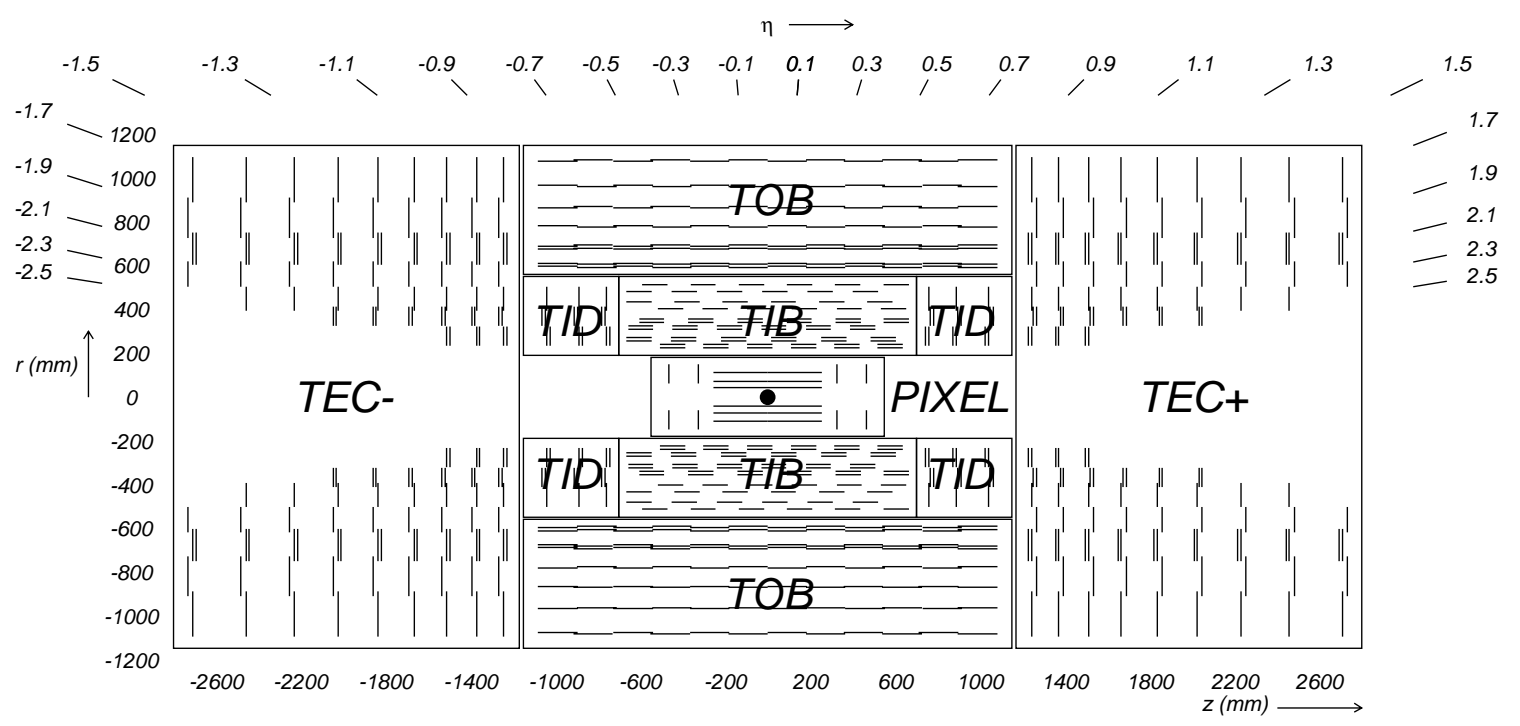

Figure 1: View of the CMS Tracker layout in the $(r, z)$ plane.

The TIB has four layers assembled in shells; the two innermost layers host double-sided detectors, built by glueing two detectors back-to-back with $100 \mathrm{mrad}$ tilted strip directions. The TID, made of three small disks with three rings each on either side, complements the TIB region. The two inner rings of each disk are equipped with double-sided modules. The outer barrel structure (TOB) consists of six concentric layers, also in this case the two innermost cylinders are equipped with double-sided modules. The TEC modules are mounted in rings on nine disks on both side of the barrel. The detectors of rings 1,2 and 5 are made of double-sided modules.

The main difference between the inner and the outer tracker modules is the thickness and dimensions of the silicon sensors. The inner tracker is made with thin sensors with $320 \mu \mathrm{m}$ thickness, $117 \mathrm{~mm}$ long strips of $64 \mathrm{~mm}$ width. The outermost modules have two daisy-chained thick sensors, with $500 \mu \mathrm{m}$ thickness, $95 \mathrm{~mm}$ long strips and $96 \mathrm{~mm}$ width. The higher thickness permits to collect a larger signal to compensate the higher noise due to longer strips. The TEC modules are divided in two categories: thick sensors for the outermost three rings, thin for the rest [4].

The total number of detector modules is 1440 for the pixel vertex detector and 15148 for the microstrip tracker, as shown in Tab. 1. A total of 88624 chips are needed to read-out the 75 million electronics channels of the 
Tracker modules. The microstrip electronics channels are more than 9 millions, while the pixel ones are about 66 millions. The large number of channels implies substantial passive material to readout, power and cool the on-detector electronics. This causes multiple scattering, nuclear interactions, electron bremsstrahlung and photon conversions, whose simulation requires an accurate evaluation of the passive material budget.

\begin{tabular}{|crcc|}
\hline \hline Subdetector & $\begin{array}{c}\text { Detector } \\
\text { Modules }\end{array}$ & $\begin{array}{c}\text { Readout } \\
\text { Chips }\end{array}$ & $\begin{array}{c}\text { Electronics } \\
\text { Channels }\end{array}$ \\
\hline Pixel Barrel (PXB) & 768 & 11520 & 47923200 \\
Pixel Forward (PXF) & 672 & 4320 & 17971200 \\
\hline PIXEL & 1440 & 15840 & 65894400 \\
\hline \hline Tracker Inner Barrel (TIB) & 2724 & 13968 & 1787904 \\
Tracker Inner Disks (TID) & 816 & 4416 & 565248 \\
Tracker Outer Barrel (TOB) & 5208 & 24192 & 3096576 \\
Tracker End-Caps (TEC) & 6400 & 30208 & 3866624 \\
\hline MICROSTRIP & 15148 & 72784 & 9316352 \\
\hline \hline TRACKER & 16588 & 88624 & 75210752 \\
\hline \hline
\end{tabular}

Table 1: Number of detector modules, readout chips and electronic channels.

The active detectors volume of the silicon Tracker is $86321 \mathrm{~cm}^{3}$ with an unprecedented value of $202 \mathrm{~m}^{2}$ of active detection surface. The weight of all the silicon sensors is $201 \mathrm{~kg}$, nearly half of this weight is due to the thick silicon microstrip sensors of the modules mounted on the outer barrel layers. The active volume, detector surface and weight of the silicon sensors are shown in Tab. 2 for each subdetector together with the partial sum for pixel and microstrips and the whole Tracker.

\begin{tabular}{|cccc|}
\hline \hline Subdetector & $\begin{array}{c}\text { Active } \\
\text { Volume } \\
{\left[\mathrm{cm}^{3}\right]}\end{array}$ & $\begin{array}{c}\text { Detector } \\
\text { Surface } \\
{\left[\mathrm{cm}^{2}\right]}\end{array}$ & $\begin{array}{c}\text { Silicon } \\
\text { Weight } \\
{[\mathrm{kg}]}\end{array}$ \\
\hline Pixel Barrel (PXB) & 215.4 & 7558.3 & 0.502 \\
Pixel Forward (PXF) & 76.5 & 2834.4 & 0.178 \\
\hline PIXEL & $292 \mathrm{~cm}^{2}$ & $1.0 \mathrm{~m}^{2}$ & $0.680 \mathrm{~kg}$ \\
\hline \hline Tracker Inner Barrel (TIB) & 5673.0 & 195621.7 & 13.218 \\
Tracker Inner Disks (TID) & 1989.2 & 68594.8 & 4.635 \\
Tracker Outer Barrel (TOB) & 42689.0 & 908275.8 & 99.465 \\
Tracker End-Caps (TEC) & 35677.3 & 833450.6 & 83.128 \\
\hline MICROSTRIP & $86029 \mathrm{~cm}^{3}$ & $200.6 \mathrm{~m}^{2}$ & $200.4 \mathrm{~kg}$ \\
\hline \hline TRACKER & $86321 \mathrm{~cm}^{3}$ & $202 \mathrm{~m}^{2}$ & $201 \mathrm{~kg}$ \\
\hline \hline
\end{tabular}

Table 2: Total active volume, detection surface and weight of the silicon detectors.

The physics analysis potential of CMS depends on the accuracy of the simulation of such a complex device. The fast shaping times of the electronics and the signal degradation due to the radiation damage caused by the large flux of particles produced in the LHC collisions are a challenge for the simulation. The accuracy of the predicted space point resolution depends on the ability to correctly model several factors: the diffusion of charges in the $4 \mathrm{~T}$ magnetic field of CMS, the delta ray emission, the inter-strip capacitance, the collected charge and the noise. In addition, a detailed description of the positioning of the detectors is demanded. 


\section{Tracker Simulation}

The simulation of the Tracker is part of the CMS software framework CMSSW [5]. It is divided into description of the detector geometry and simulation of the energy released by the charged particles crossing the silicon detectors followed by the readout of electronics signals (digitization).

\subsection{Geometry and Material Budget}

The CMS simulation software is based on Object Oriented technology using the toolkit GEANT4 [6] and CMS has chosen to provide the geometry description using the Detector Description Language (DDL) [7]. The algorithms build the volume tree as required by GEANT4 and position the volumes appropriately by providing the correct translation and rotation matrices. Each parametrisation within the DDL has a unique name, associated to a $\mathrm{C}++$ class. The shared library built with the $\mathrm{C}++$ classes is loaded on demand. Each class is often associated with a list of parameters which needs to be supplied with xml (eXtensible Markup Language) files. The $\mathrm{C}++$ class has essentially two methods, one to initialise itself by loading the parameters given in the $\mathrm{xml}$ file and the other to execute the parametrization. During the process of execution, DDL solids, logical or position parts can be created.

The design of the geometry description of the Tracker follows some rules to speed-up the parsing of the xml files and the initialization of the GEANT4 library:

- break up the content of a sub-detector into a number of sub-components;

- try to avoid duplication in defining solids or logical parts;

- use of constant names and simple expressions in the $\mathrm{xml}$ files for easy association with the mechanical components;

- use average materials in the passive part unless there are localisations of some dense material (like aluminium blocks, cooling pieces) which are then separately described in detail;

- use algorithms in positioning objects if a suitable correlation is found.

These rules make the geometrical description better readable, more easily maintainable and reusable.

The CMS Tracker geometry has been completely reviewed with the most updated information coming from the engineers' drawings of the final assembled structures. The review of the Tracker geometry consisted of two important aspects:

- check of the position and orientation of the active silicon detector volumes;

- check the correctness of the passive volumes dimensions and their materials.

The first aspect is of paramount importance to ensure the correctness of tracking, vertex reconstruction and alignment algorithms. All the Tracker active volumes are univocally identified within the CMS software framework CMSSW [5] via 32-bit integer value. This identifier is commonly called "geographical identity number" or "detector identity number", whose value is mapped with the $\mathrm{I}^{2} \mathrm{C}$ address used to electronically identify the detector modules.

The simulation of the passive volumes is performed with some simplifications to reduce the number of the volumes added to the GEANT 4 tree. Table 3 shows the number of active and passive volumes for the different parts in which the Tracker geometry is divided. The passive volumes are $95 \%$ of the total Tracker volumes and are defined using average materials.

Passive volumes material mixtures are defined from the information on the composition and measurement of the weight and dimensions of all the components (electrical and mechanical components of the electronics boards, length of the power cables, dimensions of the supporting structures, cooling fluid volume). The material properties, as the density and the radiation length, are calculated taking into account the mixture components weight fractions. A picture of the TOB cooling manifolds with the digital opto-hybrid modules, connectors, power cables and rails is shown in Fig. 2a. The corresponding description with the Tracker geometry software is shown in Fig. 2b.

The Tracker weighing procedure has been performed at first with weighing all the smallest componets and reproducing the measured weight and material composition when defining the material mixtures. Complex objects, as 


\begin{tabular}{|crrr|}
\hline \hline Subdetector & $\begin{array}{c}\text { Active } \\
\text { Volumes }\end{array}$ & $\begin{array}{c}\text { Passive } \\
\text { Volumes }\end{array}$ & $\begin{array}{c}\text { Total } \\
\text { Volumes }\end{array}$ \\
\hline Pixel Barrel (PXB) & 768 & 10201 & 10969 \\
Pixel Forward (PXF) & 672 & 23670 & 24342 \\
\hline PIXEL & 1440 & 33871 & 35311 \\
\hline \hline Inner Tracker (TIB+TID) & 3540 & 56488 & 60028 \\
Tracker Outer Barrel (TOB) & 5208 & 145419 & 150627 \\
Tracker End-Caps (TEC) & 6400 & 113158 & 119558 \\
Outer Structures & 0 & 346 & 346 \\
\hline MICROSTRIP & 15148 & 315411 & 330559 \\
\hline \hline TRACKER & 16588 & 349283 & 365871 \\
\hline \hline
\end{tabular}

Table 3: Number of GEANT4 volumes needed to construct the Tracker geometry tree.

microstrip modules, have been weighed and compared with the simulation, the measured and simulated weights compatibility is better than 5\%. Even bigger parts, as the two completed TEC endcaps and the inner tracker TIB+TID, have been weighed. The measured weights are in agreement within 5-10\% with the simulation.

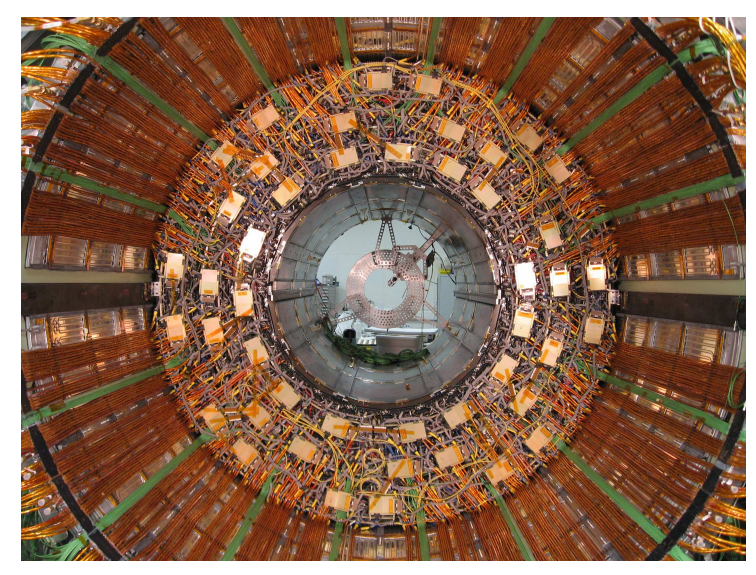

(a)

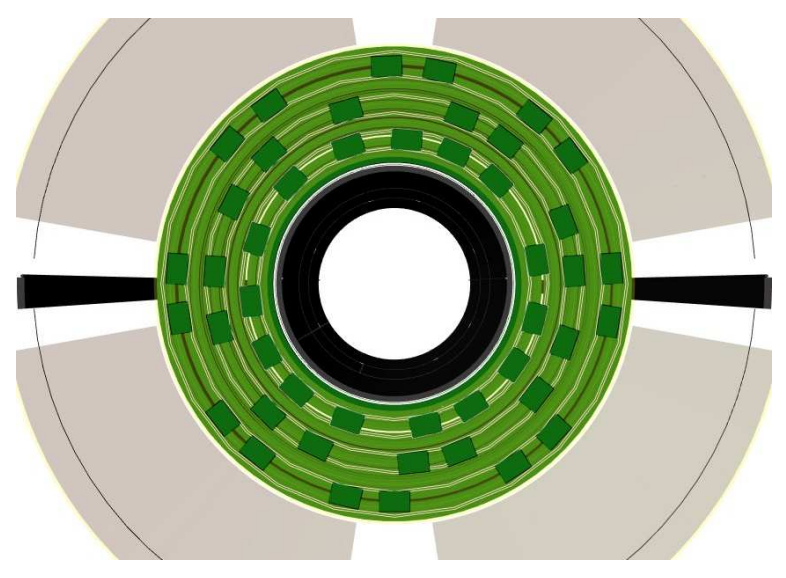

(b)

Figure 2: Photograph of the TOB services at $z>0$ with the Digital Opto-Hybrid Modules (DOHMs, the rectangles), the cooling manifolds, the connectors of the modules rods, the power cables and the rails (a) and software implementation of the same volumes within the CMSSW framework (b).

The total weight of the CMS Tracker is about 4 Tons, resulting in an average density of $0.17 \mathrm{~g} / \mathrm{cm}^{3}$. A minimum ionizing particle (mip) loses about $35 \mathrm{MeV} / \mathrm{m}$ when crossing the Tracker. The average Tracker radiation length $\mathrm{x} / \mathrm{X}_{0}$ in the barrel region $|\eta|<0.8$ is 0.4 , therefore nearly $40 \%$ of the photons will convert inside the Tracker volume. The fraction of radiation length as a function of psedorapidity, seen by particles originating from the nominal interaction point and passing straight through the Tracker, is shown in Fig. 3 for the different Tracker structures (a) or material categories (b).

The $\mathrm{x} / \mathrm{X}_{0}=1.8$ peaks near $|\eta|=1.2$ are due to the multiple crossing of the power cables: the inner Tracker radial cables at $z=800 \mathrm{~mm}$ and the axial ones at $r=500 \mathrm{~mm}$, the outer tracker radial cables placed at $z=1100 \mathrm{~mm}$ and the axial ones crossed at $r=1100 \mathrm{~mm}$, as shown in Fig. 4 .

The fraction of nuclear interaction length is below 0.6 in all $\eta$ regions, as shown on Fig. 5 for the different structures (a) or the material categories (b).

The biggest contribution to the radiation length in the Tracker is due to the support structures $(36 \%)$ and the cables to power the detector and to transport the signals through optical fibers (24\%). The electronics boards amount is $16 \%$ of the total materials and the cooling pipes and fluid $\left(\mathrm{C}_{6} \mathrm{~F}_{14}\right)$ is $14 \%$. The silicon sensitive volumes represent only $9 \%$ of the Tracker material budget. 


\section{Tracker Material Budget}

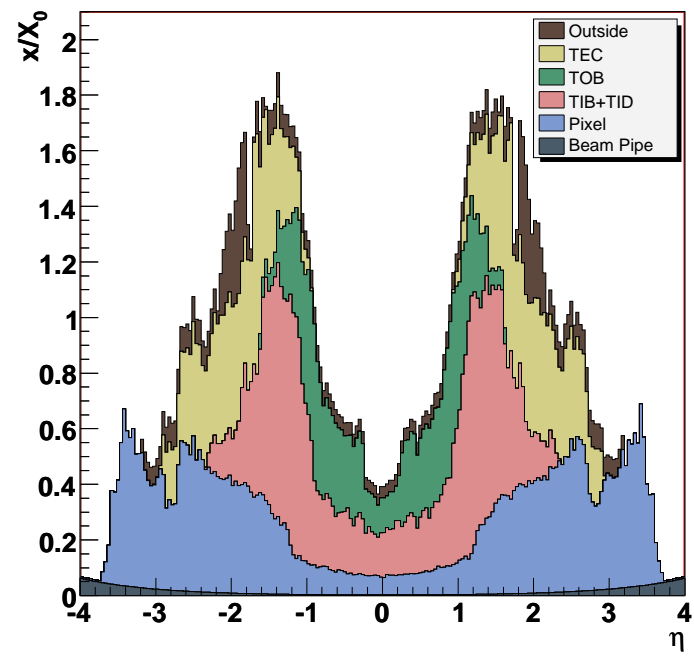

(a)

\section{Tracker Material Budget}

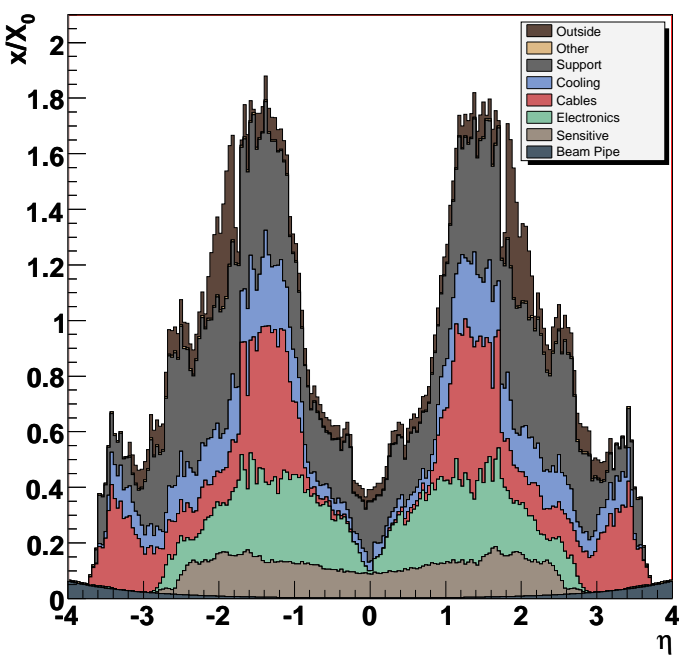

(b)

Figure 3: Material budget profile of the Tracker simulation: fraction of radiation length $\mathrm{x} / \mathrm{X}_{0}$ as a function of pseudorapidity $\eta$ (a) for the different sub-detectors and structures: the beam pipe, the pixel vertex detector, the inner Tracker (TIB+TID), the outer barrel (TOB) and endcaps (TEC), the outer structures (support tube, thermal screen and bulkheads) and (b) for the different material categories: beam pipe, silicon sensitive volumes, electronics, cables, cooling pipes and fluid, support mechanics and outer structures.

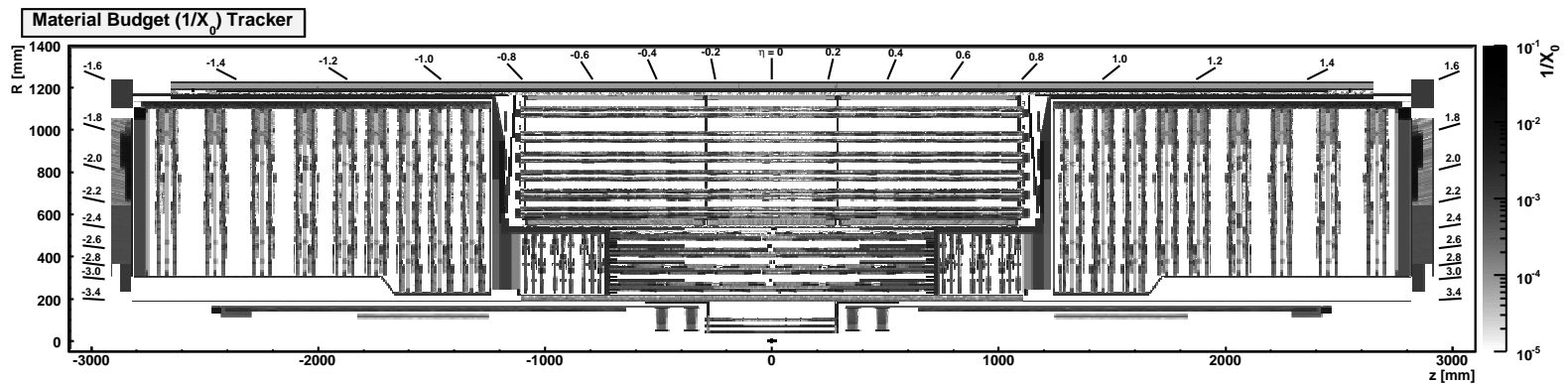

Figure 4: Material budget profile of the Tracker simulation: local amount of material $\left(1 / \mathrm{X}_{0}\right)$ in the $(r, z)$ plane. The darker regions are the ones with the larger $\mathrm{x} / \mathrm{X}_{0}$ for which the probability for a photon to convert is higher.

\subsection{Detector Response}

The particles are propagated through the Tracker volumes by GEANT4, the energy lost by the charged particles crossing the silicon active volumes is recorded together with the the entrance and the exit points in the volume. Lower thresholds for $\delta$-ray production energies are set to $30 \mathrm{keV}$ and $120 \mathrm{keV}$, for pixel and microstrip respectively, to realistically simulate the collected charge distributions. The choice of the $\delta$-ray energy thresholds has been tuned to have visible effects after the digitization and speed-up the simulation process.

The mean energy required to create an electron/hole pair in the depleted silicon is $3.6 \mathrm{eV}$. Since the most probable value of energy loss for a mip in silicon is $288 \mathrm{eV} / \mu \mathrm{m}$, in $320 \mu \mathrm{m}$ silicon thickness a mip releases 25600 electrons. This value represents an estimate of the charge collected inside an active volume. The distribution of energy released along the track segment is estimated by subdividing it into equal subsegments, small compared to the sensor pitch. Each subsegment is assigned a fraction of the deposited energy taking into account Landau fluctuations.

The charge from each subsegment is drifted toward the detector surface and simultaneously diffused in the perpendicular plane. The diffusion is distributed as a gaussian with a standard deviation proportional to the square root of the drift length with diffusion constants normalised, for $300 \mu \mathrm{m}$ thick sensors, to $2 \mu \mathrm{m}$ for the pixel and $7 \mu \mathrm{m}$ 


\section{Tracker Material Budget}

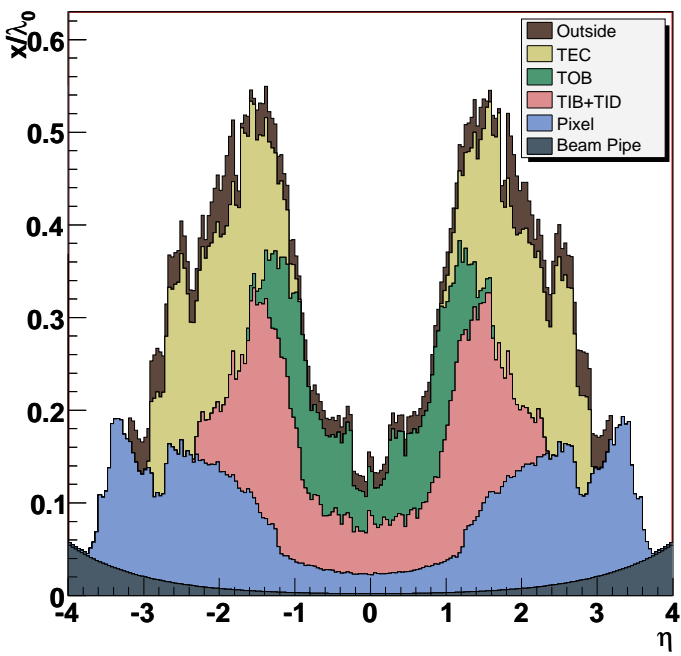

(a)

\section{Tracker Material Budget}

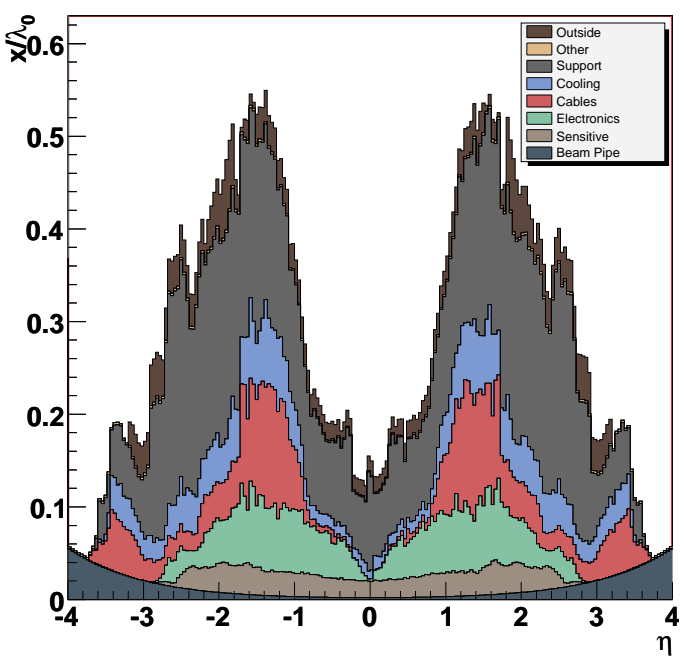

(b)

Figure 5: Material budget profile of the Tracker simulation: fraction of nuclear interaction length $\mathrm{x} / \lambda_{0}$ as a function of pseudorapidity $\eta$ (a) for the different sub-detectors and structures: the beam pipe, the pixel vertex detector, the inner Tracker (TIB+TID), the outer barrel (TOB) and endcaps (TEC), the outer structures (support tube, thermal screen and bulkheads) and (b) for the different material categories: beam pipe, silicon sensitive volumes, electronics, cables, cooling pipes and fluid, support mechanics and outer structures.

for the microstrips. The drift direction in the $4 \mathrm{~T}$ magnetic field is defined by the orientation of the sensor and the Lorentz angle, which is $23^{\circ}$ and $7^{\circ}$ for the pixel and microstrips barrel modules respectively. The different Lorentz angle is due to the different mobility of the charge carriers, electrons for the pixels and holes for the microstrips. Therefore an average $120 \mu \mathrm{m}$ charge drift is expected in a pixel sensor and between 36 and $61 \mu \mathrm{m}$ in the microstrip sensors. The resulting charge distribution is mapped to the detector geometry and the fraction of energy collected by each electronics channel is determined.

The shaping time of the microstrip readout electronics is taken into account by superimposing minimum bias collisions from the 5 preceding and 3 following LHC bunch crossings, each bunch crossing lasting 25 ns. The signals of out-of-time particles (pile-up signals) are scaled accordingly to the shape of the front-end electronics signal, shown in Fig. 6.

The pixels readout chip assigns hits to the correct bunch crossing within the $25 \mathrm{~ns}$ window, hence in the simulation only the pile-up hits from the same bunch crossing are superimposed.

At LHC design luminosity $10^{34} \mathrm{~cm}^{-2} \mathrm{~s}^{-1}$, the pixel occupancy is about $10^{-4}$, while for the microstrip it ranges from $2.5 \%$ to $0.5 \%$ from the inner to the outer layers.

The collected signals are digitized multiplying the number of electrons by a conversion factor into a 6(8)-bit ADC counts for pixel(microstrips) to mimic the electrical chain gain factor. As a result, 1 ADC count corresponds roughly to 250 electrons. A gaussian noise contribution is then added to all the channels with a standard deviation set to 500 for the pixel and 1200-1600 electrons for microstrips, depending on the strip length. This includes a conservative estimation of the noise increase with radiation damage due to the high radiation dose, $10^{14} \mathrm{n}_{\mathrm{eq}} \mathrm{cm}^{-2} \mathrm{yr}^{-1}$ after 10 years of operation at LHC. The inter-strip coupling cross-talk is simulated for the microstrip sensors assigning $6 \%$ of the charge fraction to the neighbours strips when operating in peak readout mode and $12 \%$ in deconvolution mode.

A clustering algorithm is performed both in the pixel and microstrip detectors. A pixel cluster is defined by searching for a seed with a signal-to-noise ratio greater than 6 and adding neighbour pixels, also diagonal ones, if the signal-to-noise is greater than 5. A cluster is accepted if the overall signal-to-noise ratio is greater than 10.1 . The microstrip algorithm is similar and a cluster is defined with signal-to-noise thresholds of 4 for the seed, 3 for adding the neighbouring strips and 5 to accept the cluster. 


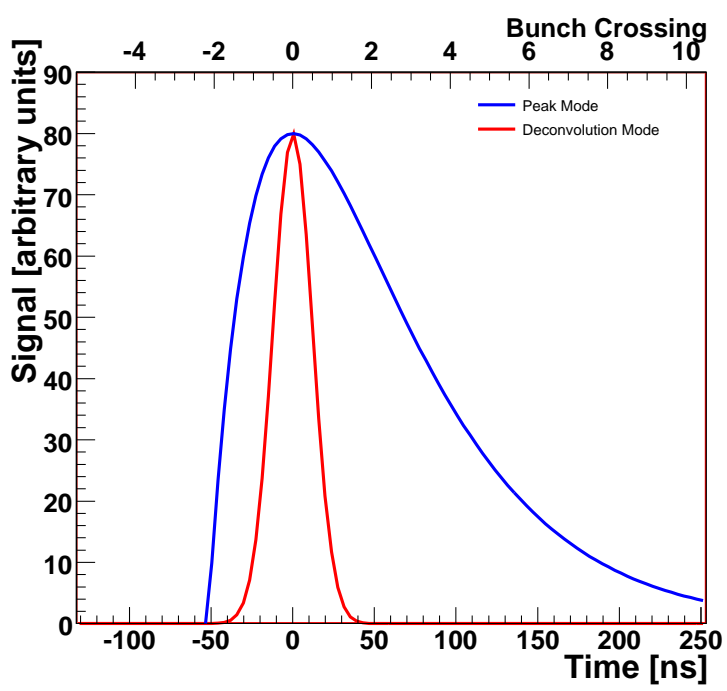

Figure 6: Evolution of the microstrip front-end signal with time, for readout mode peak or deconvolution, obtained combining together three consecutive samples to effectively reduce the signal duration (mode of operation which will be used at LHC).

The cluster finding efficiency, measured with the Monte Carlo, is greater than $99 \%$, the clusters originated by the passage of a mip in the pixel detector have a signal-to-noise ratio of about 70, while in the microstrip tracker the signal-to-noise ratio is 25 . The single-point resolution is $10 \mu \mathrm{m}$ in $r \varphi$ and $20 \mu \mathrm{m}$ along $z$ for the pixels. The microstrips resolution is $30 \mu \mathrm{m}$ in $r \varphi$ and $230 \mu \mathrm{m}$ along $z$ when interpolating the signal of the two sensors in the double-sided detectors of the inner layers. For the thick detectors of the outer layers the single-point resolution is respectively $45 \mu \mathrm{m}$ and $530 \mu \mathrm{m}[8]$.

\section{Conclusions}

The CMS all-silicon Tracker is a complex detector to construct and and requires a complex and accurate simulation. A big effort has been made to be ready for the LHC start-up, foreseen during 2008, with the best simulation of the Tracker geometry, material budget and detector response. The material budget has been estimated by measuring and weighing all the detector components and reproducing the correct composition of the materials. The detector response simulation has been tuned with data from several test beams and is continuously updated with the latest results from the analysis of cosmic ray data collected during the commissioning of the different Tracker subdetectors. 


\section{References}

[1] CMS Collaboration, “CMS: The Tracker Project Technical Design Report”, CERN/LHCC 98-06, CMS TDR 5, 15 April 1998,

CMS Collaboration, “Addendum to the CMS Tracker TDR”, CERN/LHCC 2000-016, CMS TDR 5 Addendum 1, 21 February 2000.

[2] CMS Collaboration, “The Compact Muon Solenoid Technical Proposal”, CERN/LHCC 94-38, LHCC/P1, 15 December 1994.

[3] The LHC Study Group, CERN/AC/95-05 (1995), “The Large Hadron Collider Conceptual Design Report”.

[4] L. Borrello, A. Messineo, E. Focardi and A. Macchiolo, CMS NOTE 2003/020, "Sensor design for the CMS Silicon Strip Tracker".

[5] The CMS software framework CMSSW web page is online at https://twiki.cern.ch/twiki/bin/view/CMS/CMSSW, the userguide is available at https://twiki.cern.ch/twiki/bin/view/CMS/WorkBook.

[6] Geant Collaboration, "GEANT4 - a simulation toolkit”, Nuclear Instruments and Methods in Physics Research A 506 (2003) 250-303;

Geant Collaboration, "Geant4 developments and applications", IEEE Transactions on Nuclear Science 53 No. 1 (2006) 270-278.

The GEANT4 web page is http://geant4.web.cern.ch/geant4/.

[7] M. Case and F. van Lingen, CMS NOTE-2001/057, November 2001, “Detector Description Domain Architecture \& Data Model"; http://cmsdoc.cern.ch/cms/software/ddd/www.

[8] CMS Collaboration, “CMS: Detector Performance and Software Technical Design Report”, CERN/LHCC 2006-06, CMS TDR 8.1, 2 February 2006. 